\title{
Modelo de crecimiento diamétrico de Nothofagus alpina y su relación con el de Nothofagus obliqua y Nothofagus dombeyi en los bosques naturales de la Patagonia argentina
}

\author{
Diametric growth model of Nothofagus alpina and its relationship with that of Nothofagus obliqua \\ and Nothofagus dombeyi in the natural forest from Patagonia of Argentina
}

\author{
Hernán Attis Beltrán a,b*, Luis Chauchard a,c, \\ Alejandro Dezzotti a, Guillermo Martínez Pastur ${ }^{\text {b }}$

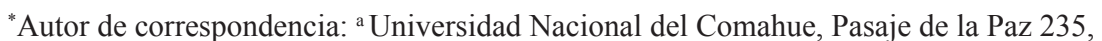 \\ Q8370AQA San Martín de Los Andes, Argentina, tel.: + 542972 427618, hattisbeltran@gmail.com \\ ${ }^{\mathrm{b}}$ Consejo Nacional de Investigaciones Científicas y Técnicas, Argentina. \\ ${ }^{\mathrm{c}}$ Administración de Parques Nacionales, Argentina.
}

\begin{abstract}
SUMMARY
Tree growth models contribute to describing the structure and functioning of forests, predicting timber production, implementing appropriate silvicultural practices and assessing the economic dimension of forestry. Nothofagus alpina, Nothofagus obliqua and Nothofagus dombeyi (Nothofagaceae) form natural forests in Northwestern Patagonia of Argentina. Models of diametric increment and yield were developed for $N$. alpina trees from the intermediate and superior canopy strata, through Bertalanffy - Richards's functions. They were interpreted together with those for $N$. obliqua and $N$. dombeyi, formerly developed using the same methodology. In all three species, intermediate trees exhibited the lowest diametric growth, probably in response to differences in the amount and composition of light. Growth of $N$. alpina trees, compared to $N$. dombeyi, was similar for all age classes within the intermediate stratum, and lower for older classes within the superior stratum. However, the performance of this species was adequate in both strata, which would be associated with the superior shade tolerance (perceived in the intermediate stratum) and the response to increasing light (in the upper one). Nothofagus obliqua experienced the slowest growth for all social strata and age classes. Discrepancies observed among models were compatible with interspecific differences in intrinsic growth and demand of light. The simultaneous interpretation of growth models of $N$. alpina, $N$. obliqua and $N$. dombeyi can contribute to deeply understand ecological patterns and processes of this subantarctic forest, aimed at its conservation and sustainable management.
\end{abstract}

Key words: subantarctic forest, timber production, crown classes, shade-tolerance.

\section{RESUMEN}

Los modelos de crecimiento arbóreo contribuyen a describir la estructura y el funcionamiento del bosque, predecir la producción de madera, implementar alternativas silvícolas y evaluar la dimensión económica forestal. Nothofagus alpina, Nothofagus obliqua y Nothofagus dombeyi (Nothofagaceae) conforman bosques naturales en el noroeste de la Patagonia argentina. A través de la función von Bertalanffy-Richards, se desarrolló un modelo de incremento corriente y rendimiento en diámetro para $N$. alpina de los estratos intermedio y superior, y se los comparó con los existentes para $N$. obliqua y $N$. dombeyi. En las tres especies, los árboles intermedios exhibieron el menor crecimiento que se debería a la composición y menor cantidad de luz. El crecimiento de $N$. alpina, comparado con el de $N$. dombeyi, fue similar en todas las clases de edad para el estrato intermedio, y menor en las clases de mayor edad para el estrato superior. Sin embargo, el desempeño de esta especie fue muy adecuado en ambos estratos, que se asociaría a la mayor tolerancia a la sombra (percibida en el estrato intermedio) y la respuesta al aumento de la luz (en el superior). Nothofagus obliqua siempre presentó el crecimiento diamétrico más lento. Las discrepancias observadas en los modelos fueron compatibles con diferencias interespecíficas de crecimiento intrínseco y demanda de luz. La interpretación simultánea de los modelos de estas especies de Nothofagus contribuye a aumentar la comprensión ecológica y productiva de un valioso tipo de vegetación subantártica.

Palabras clave: bosque subantártico, producción forestal, estratos sociales, tolerancia a la sombra.

\section{INTRODUCCIÓN}

Los modelos de crecimiento arbóreo representan una herramienta cuantitativa valiosa para la gestión sostenible porque contribuyen a describir la estructura y el funcionamiento del bosque, predecir la producción de madera, implementar alternativas silvícolas y evaluar la dimensión económica a lo largo de gradientes de edad, calidad de si- 
tio y uso forestal. Estos modelos pueden comprender ecuaciones relativamente simples lo que facilita enormemente su aplicación (Vanclay 1994). El crecimiento de una planta representa la variación del tamaño y peso a lo largo del tiempo, e integra los efectos de patrones y procesos físicoquímicos y ecofisiológicos tales como la fotosíntesis, la competencia y la asignación de recursos a las partes aéreas y subterráneas (Smith et al. 1996). La luz es un componente físico clave del crecimiento de una planta debido a los efectos directos e indirectos sobre el desempeño individual (Pallardy 2007). La respuesta de las especies arbóreas a la variación de luz es continua; sin embargo, se reconocen clases discretas de tolerancia a la sombra que permiten explicar aspectos fundamentales de la sucesión ecológica de los bosques naturales (Tilman 1988).

El diámetro del fuste del árbol es una dimensión clave de los modelos de producción forestal que se mide en forma sencilla, se relaciona con el tamaño de la copa, el área basal y el volumen del tronco, permitiendo desarrollar diagramas de manejo de la densidad del bosque (Smith et al. 1996). En particular, los modelos biológicos del crecimiento diamétrico individual se emplean para interpretar en forma causal patrones y procesos ecosistémicos en el corto plazo (Vanclay 1994). El diámetro tiende a seguir una trayectoria sigmoidea variable a lo largo del tiempo que depende de las características intrínsecas de la especie, la edad del individuo, la densidad y cobertura poblacional así como las propiedades físicas y químicas del sitio. La interacción de estos factores origina la diferenciación social manifestada en las clases de copa (Smith et al. 1996).

El bosque alto y cerrado compuesto por las especies caducifolias Nothofagus alpina (Poepp. et Endl.) Oerst. (raulí) y Nothofagus obliqua (Mirb.) Oerst. (roble pellín), y la siempreverde Nothofagus dombeyi (Mirb.) Oerst. (coihue) (Nothofagaceae), se desarrolla en los faldeos de las cordilleras de La Costa y Los Andes, entre $39^{\circ} 29^{\prime}$ y $40^{\circ} 22^{\prime} \mathrm{S}$ y hasta 1.000 m s.n.m. (Lara et al. 1999). Estos árboles son diclino monoicos y anemocoros, y experimentarían una producción anual variable de semillas que conforman un banco transitorio en el suelo (Dezzotti et al. 2016). Las especies de Nothofagus desarrollan poblaciones coetáneas luego de la ocurrencia de disturbios de gran escala. Las perturbaciones de pequeña escala promueven un bosque en mosaico, formado por parches cuya estructura depende del tamaño y la forma de los claros y el comportamiento ecológico de las especies (Veblen et al. 1996).

Los renovales de $N$. alpina, $N$. obliqua y $N$. dombe$y i$ tienen un modo de regeneración común dependiente de claros del dosel; sin embargo, estas especies presentan divergencias en la sobrevivencia, el crecimiento y el desarrollo bajo diferentes regímenes de luz. La diferenciación del nicho de regeneración explicaría la variación composicional y estructural de este tipo forestal a lo largo del espacio y el tiempo (Veblen et al. 1996). En particular, $N$. alpina es la especie más tolerante a la sombra, a juzgar por la capacidad de los estadios iniciales de persistir como "regeneración avanzada" y crecer más rápidamente en condiciones sombrías. Esta capacidad se vincula en parte al mayor tamaño y acumulación de nutrientes de la semi1la (Read y Hill 1985, Grosse 1988, Ramírez et al. 1997, Weinberger y Ramírez 2001, Dezzotti et al. 2004).

Los objetivos de este estudio son: i) desarrollar un modelo individual de incremento corriente y rendimiento diamétrico de árboles de $N$. alpina del estrato intermedio y superior, e ii) interpretar este modelo en forma simultánea con los de $N$. dombeyi y $N$. obliqua, desarrollados en la misma área de estudio y con una metodología equivalente (Chauchard et al. 2001, Chauchard y Sbrancia 2003). La hipótesis que se busca validar propone que existe un patrón de crecimiento diamétrico común de $N$. alpina, $N$. obliqua y $N$. dombeyi, pero al mismo tiempo subsisten discrepancias interespecíficas en cuanto a las magnitudes de crecimiento asociadas a la tolerancia a la sombra. La interpretación conjunta de los modelos de crecimiento de las especies de Nothofagus puede contribuir a lograr una mayor comprensión de la ecología y productividad de este valioso bosque Subantártico en el contexto del manejo sostenible.

\section{MÉTODOS}

Área de estudio. El modelo de crecimiento para N. alpi$n a$ se basó en datos colectados en las estaciones de muestreo Quilanlahue ( $\left.40^{\circ} 09^{\prime} \mathrm{S}, 71^{\circ} 28^{\prime} \mathrm{O}\right)$, Yuco $\left(40^{\circ} 09^{\prime} \mathrm{S}\right.$, $\left.71^{\circ} 31^{\prime} \mathrm{O}\right)$, Hua hum ( $\left.40^{\circ} 09^{\prime} \mathrm{S}, 71^{\circ} 35^{\prime} \mathrm{O}\right)$, Nonthué ( $40^{\circ} 07^{\prime} \mathrm{S}, 71^{\circ} 38^{\prime} \mathrm{O}$ ) y Pucará ( $40^{\circ} 09^{\prime} \mathrm{S}, 71^{\circ} 39^{\prime} \mathrm{O}$ ) (Parque Nacional Lanín, Argentina). En esta región, el clima es templado húmedo con un pronunciado gradiente de precipitación longitudinal, provocado por la sombra de lluvias que ejerce la cordillera de los Andes, y estacional, debido al desplazamiento anual del centro de presión del océano Pacífico (Paruelo et al. 1998). En el área Quechuquina $\left(40^{\circ} 09^{\prime} \mathrm{S}, 71^{\circ} 34^{\prime} \mathrm{O}, 1965\right.$ - 2009), circundante a las estaciones de muestreo, la temperatura media y la precipitación total anuales son $9,3{ }^{\circ} \mathrm{C}$ y $1.889 \mathrm{~mm}$, respectivamente $^{1}$ (figura 1). Los suelos son Andisoles con un perfil $\mathrm{O}-\mathrm{A}-\mathrm{B}_{\mathrm{w}}-\mathrm{C}$, tienen abundante cantidad de limo, arcilla y materia orgánica, y alta capacidad de retención hídrica, intercambio catiónico y fijación de fósforo (del Valle 1998).

La vegetación pertenece al Distrito del Bosque Caducifolio de la Provincia Subantártica (Cabrera 1971). Las estaciones de muestreo se instalaron en áreas preservadas de perturbaciones antrópicas recientes, intensas y de gran escala, y representaron parte de la heterogeneidad estructural de los rodales presentes (APN 2012). Estas estaciones estaban ocupadas por bosques regulares en diferentes fases de desarrollo (sensu Oliver y Larson 1996), a juzgar por la forma normal de la distribución de tallas, y las

\footnotetext{
Dirección Provincial de Bosques de Neuquén, Argentina, datos no publicados.
} 
relaciones entre la densidad y el volumen del rodal y el diámetro de los individuos. Los rodales jóvenes presentaron mayor densidad y menores volumen y diámetro (fase de exclusión), mientras que los maduros menor densidad y mayores volumen y diámetro (fase de bosque maduro) (Dezzotti et al. 2016) (cuadro 1).

Colección de datos. Los datos para ajustar el modelo de crecimiento de $N$. alpina provinieron de árboles adultos (diámetro a la altura del pecho a $1,3 \mathrm{~m}$, dap $\geq 10 \mathrm{~cm}$ ), localizados en bosques puros, y mixtos con $N$. obliqua y $N$. dombeyi, con cobertura de copas continua, altura máxima entre 25 y $35 \mathrm{~m}$ y sin extracción de madera durante el ciclo de desarrollo del rodal (Attis Beltrán et al. 2015). Las muestras se colectaron de individuos en pie presentes en parcelas permanentes de muestreo y de apeados fuera de ellas. Estas parcelas fueron circulares de $500(\mathrm{n}=23)$ y $1.000 \mathrm{~m}^{2}(\mathrm{n}=6)$, y cuadradas de $2.000 \mathrm{~m}^{2}$ cada una $(\mathrm{n}=4)$. A los árboles presentes en las parcelas se los etiquetó con un número, y se les señaló la circunferencia del tronco (pintura), midió el dap (cinta diamétrica) al inicio y final de un período de entre cuatro y nueve años y asignó la clase de dap (clase 1: 10,1 - 15,0; clase 2: 15,1 - 20,0;..; clase $11: \geq 60,1 \mathrm{~cm})$ y el estrato social.

Los estratos sociales fueron i) dominante: árbol con copa mayor en referencia a la del dosel superior y que recibe luz directa en la parte superior y lateral, ii) codominante: árbol con abundante ramas y follaje, copa menor a la dominante y que recibe luz directa en la parte superior y lateral, e iii) intermedio: árbol con abundante follaje, copa pequeña y comprimida lateralmente y que recibe luz di- recta solo en la parte superior. Los árboles oprimidos, con copa pequeña que no recibe luz directa, no se analizaron. En cada parcela permanente de muestreo se eligió en forma aleatoria un árbol de cada clase de tamaño y estrato social y se lo barrenó a 1,3 m del suelo (barreno de Pressler). La base de datos se completó incluyendo árboles con estas características localizados fuera de las parcelas, los cuales se apearon y se les extrajo una rodela a $1,3 \mathrm{~m}$ del suelo $(\mathrm{n}=57)$. Los anillos de crecimiento de los tarugos y las rodelas se contaron y midieron para estimar la edad y el incremento anual y periódico de los últimos 5 y 10 años (calibre, regla y lupa de $10 \mathrm{x}$ ), a través de la metodología de Stokes y Smiley (1996).

Modelo de crecimiento diamétrico. El modelo de crecimiento individual de $N$. alpina se ajustó a partir de la relación de la edad a la altura del pecho ( $t$, años) y el incremento diamétrico corriente $\left(i d, \mathrm{~cm}\right.$ año $\left.\mathrm{o}^{-1}\right)$, y el dap y estrato social de los árboles $(\mathrm{n}=195)$. En primer lugar, se analizó estadísticamente la relación entre dap e incremento diamétrico corriente para cada clase de dap y estrato social. Los estratos sociales dominante y codominante se combinaron en el estrato superior (ES), porque los pares de datos de dap e incremento diamétrico corriente se solaparon (figura 2). La fusión de los estratos dominante y codominante fue realizada para $N$. dombeyi (Chauchard et al. 2001) y N. obliqua (Chauchard y Sbrancia 2003).

La variación del incremento diamétrico corriente con el tamaño del tronco para $N$. alpina se expresó a través de la función biológica no lineal von Bertalanffy - Richards (Richards 1959, Vanclay 1994). Este modelo de crecimien-



Figura 1. Localización de las estaciones de muestreo Quilanlahue, Yuco, Hua hum, Nonthué y Pucará, y la estación meteorológica Quechuquina en la cuenca de los lagos Lácar y Nonthué en el noroeste de la provincia de Neuquén (Argentina).

Location of the sampling sites Quilanlahue, Yuco, Hua hum, Nonthué and Pucará, and the meteorological station Quechuquina within the Lácar and Nonthué watershed in the Northwestern region of Neuquén province (Argentina). 
Cuadro 1. Estructura de rodales de Nothofagus spp. alpina $(\mathrm{Na})$, N. obliqua $(\mathrm{No})$ y $N$. dombeyi $(\mathrm{Nd})$ en cuatro estaciones de muestreo del área de estudio. $D$ : densidad (árboles ha-1), $A B$ : área basal $\left(\mathrm{m}^{2} \mathrm{ha}^{-1}\right), V$ : volumen $\left(\mathrm{m}^{3} \mathrm{ha}^{-1}\right)$, dap $:$ diámetro cuadrático medio $(\mathrm{cm})$, $h_{\text {máx: }}$ : altura máxima (m) y $c c$ : cobertura de copas (\%). Se indica además la cantidad de árboles sanos $(S)$, bien formados $(B F)$, dominantes $(D M)$ y codominantes $(C D)$ (Dezzotti et al. 2016).

Structure of four stands of $N$. alpina $(\mathrm{Na}), N$. obliqua $(\mathrm{No})$ and $N$. dombeyi $(\mathrm{Nd})$ located within the study area. $\mathrm{D}$ : density (individuals ha $\left.{ }^{-1}\right)$, $A B$ : basal area $\left(\mathrm{m}^{2} \mathrm{ha}^{-1}\right), V$ : volume $\left(\mathrm{m}^{3} \mathrm{ha}^{-1}\right)$, dap $p_{c}$ : mean quadratic diameter $\left(\mathrm{cm}\right.$ individual $\left.{ }^{-1}\right), h_{\text {máx }}:$ maximum tree height $\left(\mathrm{m}\right.$ individual $\left.{ }^{-1}\right)$ and $c c$ : $\mathrm{crown}$ cover (\%). Number of healthy $(S)$, well formed $(B F)$, dominant $(D M)$ and co-dominant trees $(C D)$ are also indicated (Dezzotti et al. 2016).

\begin{tabular}{|c|c|c|c|c|c|c|c|c|}
\hline \multirow{2}{*}{ Estación } & \multirow{2}{*}{ Variable } & \multicolumn{6}{|c|}{ Especie } & \multirow{2}{*}{ Total / Promedio } \\
\hline & & N. alpina & $\%$ & N. obliqua & $\%$ & N. dombeyi & $\%$ & \\
\hline \multirow{10}{*}{$\begin{array}{l}\text { Quilanlahue } \\
40^{\circ} 08^{\prime}, 60^{\prime \prime} \mathrm{S} \\
71^{\circ} 28^{\prime} 00^{\prime \prime} \mathrm{O} \\
750 \text { m s.n.m. }\end{array}$} & $D$ & & & 80 & 42 & 110 & 58 & 190 \\
\hline & $A B$ & & & 13,9 & 28 & 35,0 & 72 & 48,9 \\
\hline & $V$ & & & 173,8 & 16 & 926,1 & 84 & 1.100 \\
\hline & $\operatorname{dap}_{c}$ & & & 47,0 & & 63,7 & & 55,4 \\
\hline & $h_{\max }$ & & & 40,7 & & 38,0 & & 39,4 \\
\hline & $c c$ & & & 18,1 & 22 & 52,8 & 66 & 70,9 \\
\hline & $S$ & & & & 26,3 & & 36,8 & 31,6 \\
\hline & $B F$ & & & & 26,3 & & 21,1 & 23,7 \\
\hline & $D M$ & & & & 5,3 & & 26,3 & 15,8 \\
\hline & $C D$ & & & & 10,5 & & 5,3 & 7,9 \\
\hline \multirow{10}{*}{$\begin{array}{l}\text { Yuco Alto Oriental } \\
40^{\circ} 08^{\prime} 52^{\prime \prime} \mathrm{S} \\
71^{\circ} 29^{\prime} 46^{\prime \prime} \mathrm{O} \\
950 \text { m s.n.m. }\end{array}$} & $D$ & 50 & 45 & 20 & 18 & 40 & 36 & 110 \\
\hline & $A B$ & 7,6 & 11 & 10,9 & 16 & 50,6 & 73 & 69,1 \\
\hline & V & 92,3 & 10 & 177,0 & 19 & 656,7 & 71 & 926 \\
\hline & $d a p_{c}$ & 43,6 & & 83,1 & & 127,0 & & 84,6 \\
\hline & $h_{\max }$ & 27,2 & & 32,0 & & 39,1 & & 32,8 \\
\hline & $c c$ & 9,7 & 11 & 15,3 & 18 & 50,0 & 58 & 75,0 \\
\hline & $S$ & & 36,4 & & 0,0 & & 18,2 & 18,2 \\
\hline & $B F$ & & 9,1 & & 18,2 & & 0,0 & 9,1 \\
\hline & $D M$ & & 0,0 & & 0,0 & & 18,2 & 6,1 \\
\hline & $C D$ & & 0,0 & & 18,2 & & 18,2 & 12,1 \\
\hline \multirow{10}{*}{$\begin{array}{l}\text { Yuco Alto Occidental } \\
40^{\circ} 08 \text { “9”' S } \\
71^{\circ} 30 \text { ' } 19 \text { ” O } \\
844 \text { m s.n.m. }\end{array}$} & $D$ & 40 & 9 & 380 & 83 & 40 & 9 & 460 \\
\hline & $A B$ & 5 & 9 & 42,8 & 73 & 10,5 & 18 & 58,3 \\
\hline & V & 57,9 & 7 & 450,5 & 57 & 283,4 & 36 & 792 \\
\hline & $d a p_{c}$ & 39,8 & & 37,8 & & 57,8 & & 45,1 \\
\hline & $h_{\text {máx }}$ & 34,2 & & 34,7 & & 37,0 & & 35,3 \\
\hline & $c c$ & 11,1 & 13 & 68,1 & 78 & 8,3 & 9 & 87,5 \\
\hline & $S$ & & 6,5 & & 80,4 & & 4,3 & 30,4 \\
\hline & $B F$ & & 4,3 & & 54,3 & & 2,2 & 20,3 \\
\hline & $D M$ & & 0,0 & & 4,3 & & 2,2 & 2,2 \\
\hline & $C D$ & & 6,5 & & 32,6 & & 2,2 & 13,8 \\
\hline \multirow{10}{*}{$\begin{array}{c}\text { Pucará } \\
40^{\circ} 09^{\prime} 50^{\prime \prime} \mathrm{S} \\
71^{\circ} 39^{\prime} 00^{\prime \prime} \mathrm{O} \\
700 \text { m s.n.m. }\end{array}$} & $D$ & 30 & 30 & & & 70 & 70 & 100 \\
\hline & $A B$ & 19,5 & 33 & & & 39,2 & 67 & 58,7 \\
\hline & V & 271,1 & 20 & & & $1.071,0$ & 80 & 1.342 \\
\hline & $d a p_{c}$ & 91,0 & & & & 84,4 & & 87,7 \\
\hline & $h_{\max }$ & 35,8 & & & & 45,0 & & 40,4 \\
\hline & $c c$ & 18,1 & 23 & & & 54,2 & 70 & 72,3 \\
\hline & $S$ & & 20,0 & & & & 30,0 & 25,0 \\
\hline & $B F$ & & 20,0 & & & & 0,0 & 10,0 \\
\hline & $D M$ & & 20,0 & & & & 20,0 & 20,0 \\
\hline & $C D$ & & 10,0 & & & & 40,0 & 25,0 \\
\hline
\end{tabular}




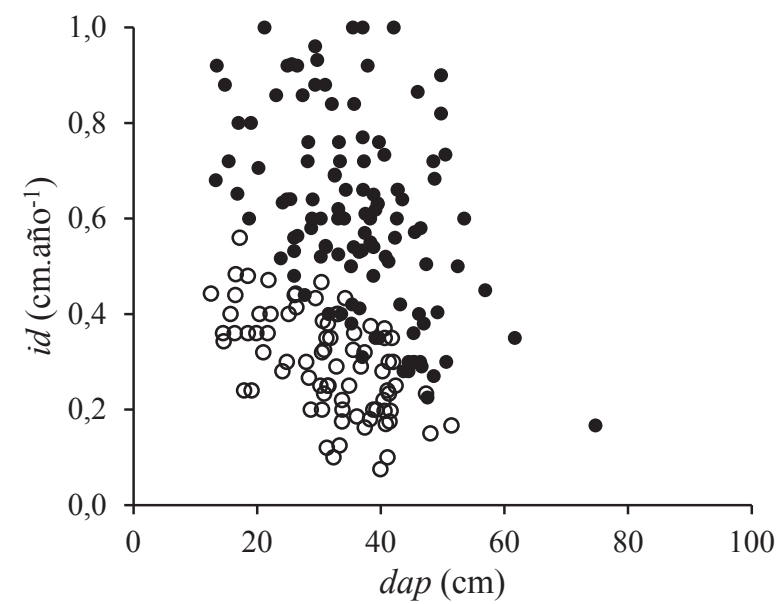

Figura 2. Incremento diamétrico corriente (id) y diámetro a la altura del pecho (dap) de Nothofagus alpina de los estratos intermedio (círculo blanco, $\mathrm{n}=78$ ) y superior (círculo negro, $\mathrm{n}=117$ ).

Current diametric increment $(i d)$ and diameter at breast height (dap) of N. alpina of the suppressed (square, $\mathrm{n}=22$ ), intermediate (white circle, $n=78)$ and superior strata $(n=117)$.

to individual expresa la variación de tamaño de árboles que no cambian de estrato social a lo largo del ciclo de vida. Sin embargo, los árboles en forma espontánea o por efecto de la silvicultura, pueden atravesar etapas de supresión y liberación en función de las características intrínsecas de la especie, la edad y la ocupación y las propiedades físicas del sitio (Smith et al. 1996). Sin embargo, esta posibilidad no afectaría la capacidad del modelo para predecir el incremento corriente y rendimiento diamétricos de un determinado estrato social durante un período limitado (Vanclay 1994). La expresión diferencial del modelo denominado Bernoulli es:

$$
\frac{d y}{d t}=a y^{c}-b y
$$

donde, $d y / d t$ : incremento instantáneo, $a$ y $b$ : constantes de anabolismo y catabolismo, respectivamente, $y$ : tamaño parcial o total individual o poblacional (en el presente estudio dap) y $c$ : constante de alometría (para $0<c<1 \Rightarrow a$, $b>0$; para $c>1 \Rightarrow a, b<0$ ).

La iniciación del análisis del crecimiento individual a partir de la ecuación [1], por un lado, no requiere conocer la edad de los árboles -cuya determinación demanda un esfuerzo considerable particularmente en bosques naturales- y, por el otro lado, permite obtener la variación del tamaño de los árboles a lo largo el tiempo (Tomé et al. 2006). La ecuación [1] se reformuló según Chauchard et al. (2001) a la forma:

$$
i d=a\left(\operatorname{dap}^{c}-\operatorname{dap} \operatorname{dap}_{m}{ }^{c-1}\right)
$$

donde, $d a p_{m}$ : diámetro máximo asintótico $(\mathrm{cm})$ representa el diámetro máximo que un árbol de un estrato determinado puede alcanzar naturalmente.

Para evaluar la bondad del ajuste no lineal se utilizaron la suma de cuadrados residuales, el error estándar, el error absoluto medio y el coeficiente de determinación. Además, el ajuste de la función se aceptó cuando el nivel de significancia de los parámetros biológicos describió el comportamiento de la especie de acuerdo a valores esperados. En general, los valores iniciales de los parámetros de la función de regresión no lineal provinieron de Chauchard (1991). En cambio, dap $p_{\mathrm{m}}$ se obtuvo de la distribución diamétrica de cada estrato obtenido de las parcelas de inventario de rodales sin intervención silvicultural.

En segundo lugar, se obtuvo la variación del dap a lo largo del tiempo a partir de la solución de la ecuación [1], de acuerdo a:

$$
d a p=d a p_{m}\left(1-e^{(-k t)}\right)^{\frac{1}{1-c}}
$$

donde, $k$ : parámetro. Esta función es sigmoidea con una asíntota superior $d a p_{m}$ y un punto de inflexión dependiente de $c(0<c<1)$.

En tercer lugar, se obtuvo el incremento diamétrico corriente ( $i d$ ) en función de $t$ a partir de la forma derivada de la ecuación [2], de acuerdo a:

$$
i d=\frac{k}{1-c} \operatorname{dap}_{m}\left(1-e^{(-k t)}\right)^{\left(\frac{1}{1-c}\right)-1} e^{(-k t)}
$$

La ecuación [2] se utilizó para realizar el ajuste estadístico porque explicitó la relación entre el incremento diamétrico corriente y $d_{a p}$ (Chauchard et al. 2001). El modelo desarrollado es consistente para árboles con una edad aproximada de hasta 200 años, teniendo en cuenta que el $d a p_{m}$ de individuos del estrato intermedio fue $50 \mathrm{~cm}$ y del estrato superior fue $75,5 \mathrm{~cm}$.

A partir del modelo von Bertalanffy - Richards se estimaron los siguientes parámetros de vida, que permitieron una mejor comprensión del crecimiento individual (Richards 1959, Melo y Vargas 2003):

$$
\begin{gathered}
c m a=\frac{d a p_{m} k}{2 c+2} \\
t v=\frac{2 c+2}{k} \\
d p i=\operatorname{dap}_{m} c^{\frac{1}{1-c}}
\end{gathered}
$$




$$
\begin{gathered}
c p i=\frac{k}{m} \\
t p i=\frac{1}{k} \ln (1-c)
\end{gathered}
$$

donde, $c m a$ : crecimiento medio absoluto $\left(\mathrm{cm} \cdot \mathrm{año}^{-1}\right), t v$ : tiempo de vida requerido para alcanzar $d a p_{m}$, (años), $d p i$ : diámetro en el punto de inflexión $(\mathrm{cm})$, crpi: crecimiento relativo en el punto de inflexión (\%) y tpi: tiempo en el que ocurre el máximo incremento corriente anual en el punto de inflexión de la función (años).

El incremento y rendimiento diamétricos de $N$. alpina se los comparó con los de $N$. dombeyi y $N$. obliqua, obtenidos por Chauchard et al. (2001) y Chauchard y Sbrancia (2003).

La comparación interespecífica dentro de cada estrato social se basó en el análisis, por un lado, visual de las curvas de los modelos de incremento corriente y rendimiento diamétricos y, por otro lado, de la expresión proporcional del dap e incremento diamétrico corriente respecto de la especie que alcanzó el valor máximo de estas variables. La existencia de diferencias significativas de las medias de las diferentes variables, entre estratos sociales, tamaños de árboles y especies, se evaluaron con el análisis de la varianza (ANDEVA) porque los datos cumplieron con los supuestos de la evaluación paramétrica referidos a la homogeneidad de la varianza y la normalidad del error $(P<0,05)$.

\section{RESULTADOS}

El modelo de crecimiento de N. alpina. El incremento diamétrico corriente promedio del estrato inferior fue $0,30 \mathrm{~cm}$ año ${ }^{-1}(\mathrm{EE}=0,01 ;$ mín. 0,08 y máx. 0,$56 ; \mathrm{n}=78)$ y del estrato superior fue $0,61 \mathrm{~cm}_{\text {año }}{ }^{-1}(\mathrm{EE}=0,02 ;$ mín. $0,17 \mathrm{y}$ máx. 1,$05 ; \mathrm{n}=117$ ); estos valores difirieron significativamente entre sí (ANDEVA, $\mathrm{F}_{1,194}=157,5 ; P<0.0001$ ). En ambos estratos, el valor de incremento diamétrico corriente disminuyó en las clases de mayor tamaño. En el estrato inferior, el incremento diamétrico máximo $\left(i d_{\text {maxx }}\right)$ fue $0,39 \mathrm{~cm} \cdot$ año $^{-1}$ en la clase $10,1-15,0 \mathrm{~cm}$ dap y el incremento diamétrico mínimo $\left(\mathrm{id}_{\min }\right.$ ) fue $0,18 \mathrm{~cm}$ año ${ }^{-1}$ en la clase $45,1-50,0 \mathrm{~cm}$ dap. En el estrato superior $i d_{\max }$ fue $0,83 \mathrm{~cm}$ año ${ }^{-1}$ en la clase $10,1-15,0 \mathrm{~cm}$ dap e $i d_{\min }$ fue $0,17 \mathrm{~cm} \cdot \mathrm{año}^{-1}$ en la clase $\geq 60,1 \mathrm{~cm}$ dap. El valor de incremento diamétrico corriente difirió entre clases de tamaño dentro de cada estrato social (ANDEVA, $P<0,05$ ) (figura 3).

En $N$. alpina, la función $i d=f($ dap $)$ exhibió valores de cuadrado medio del error, error estándar, error absoluto medio y coeficiente de determinación para el estrato superior iguales a $0,00016,0,009,0,012$ y 0,996 , respectivamente $(\mathrm{n}=117)$, y para el estrato inferior $0,00004,0,007$, 0,004 y 0,994 , respectivamente $(n=78)$. La comparación de los valores de los parámetros del modelo de incremento en diámetro entre $N$. alpina, $N$. obliqua y $N$. dombeyi se in-

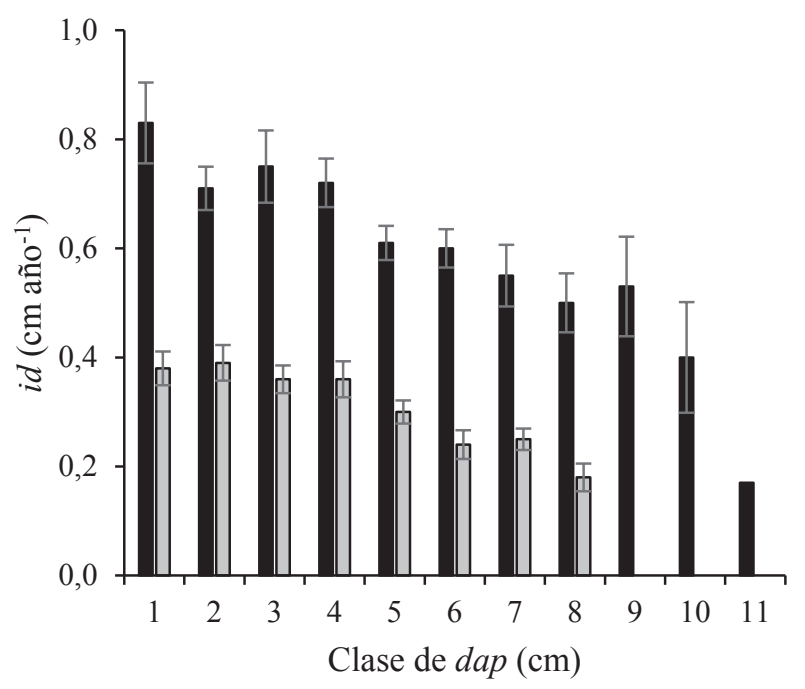

Figura 3. Incremento diamétrico corriente para Nothofagus alpina (id, $\mathrm{cm}$ año ${ }^{-1}$ ) del estrato intermedio (barra blanca, $\mathrm{n}=78$ ) y superior (barra negra, $\mathrm{n}=117$ ) con relación al diámetro a la altura del pecho (dap, clase 1: 10,1 - 15,0; clase 2: 15,1 - 19,9;..; clase $11: \geq 60,1 \mathrm{~cm})$. La línea vertical y el número indican el error estándar de la media y el tamaño de la muestra, respectivamente. id difiere entre clases de tamaño dentro del estrato social (ANDEVA, $P<0,05$ ).

Current diametric increment of $N$. alpina $\left(i d, \mathrm{~cm}_{\text {year }}{ }^{-1}\right)$ of the intermediate (white bar, $\mathrm{n}=78$ ) and superior strata (black bar, $\mathrm{n}=117$ ) in relation to diameter at breast height (dap) (class 1: $10.1-15.0$, class 2: $15.1-19.9, \ldots$, class 11: $>60.1 \mathrm{~cm})$. The vertical line and number of bars indicate the standard error of the mean and sample size, respectively. id differs among size classes within social strata (ANOVA, $P<0.05$ ).

dica en el cuadro 2. En el estrato inferior, el $i d_{\text {máx }}$ fue igual a $0,41 \mathrm{~cm}$ año $0^{-1}$ y se produjo en la clase $10,1-15,0 \mathrm{~cm}$ dap, mientras que el valor nulo de incremento diamétrico corriente se produjo en la clase $75,1-80,0 \mathrm{~cm}$. En el estrato superior el $i d_{\text {max }}$ fue igual a $0,76 \mathrm{~cm}$ año ${ }^{-1}$ y se produjo en la clase 15,1 - 20,0 cm dap, mientras que el valor nulo de incremento diamétrico corriente se produjo en la clase 90,1 - 95,0 cm dap (figura 4).

En $N$. alpina, la función dap $=f(t)$ estimó a los 5, 50, 100,150 y 200 años un valor de dap para el estrato inferior de $1,3,18,9,35,6,47,4$ y $55,4 \mathrm{~cm}$, respectivamente, y para el estrato superior $2,5,34,6,59,4,73,3$ y $80,7 \mathrm{~cm}$, respectivamente. Estos valores representaron un rendimiento del estrato superior mayor al del estrato inferior entre $90 \%$ (en los individuos más jóvenes) y $50 \%$ (en los de mayor edad) (figura 5). La función $i d=f(t)$ estimó una variación del valor de incremento diamétrico corriente para el estrato inferior entre 0,32 (5 años) y $0,13 \mathrm{~cm}^{2}$ año $^{-1}$ (200 años), y para el estrato superior entre 0,62 ( 5 años) y $0,11 \mathrm{~cm}_{\text {año }}{ }^{-1}$ (200 años). El $i d_{\text {máx }}$ para el estrato inferior fue $0,41 \mathrm{~cm}^{2}$ año${ }^{1}$ a los 30 años, y para el estrato superior $0,76 \mathrm{~cm}$ año $0^{-1}$ a los 20 años. El valor de incremento diamétrico corriente del estrato superior fue entre un $90 \%$ (en los individuos 
Cuadro 2. Valores absoluto y comparado entre especies y estratos (EI: intermedio, ES: superior) de los parámetros del modelo de incremento en diámetro para N. alpina (Na), N. obliqua (No) (Chauchard y Sbrancia 2003) y N. dombeyi (Nd) (Chauchard et al. 2001). $a, k, c$ : parámetros de vida, dap $p_{m}$ : diámetro máximo asintótico $(\mathrm{cm}), c m a$ : crecimiento absoluto medio $\left(\mathrm{cm}\right.$ año $\left.{ }^{-1}\right), t v$ : tiempo de vida requerido para alcanzar $d a p_{m}$ (años), dpi: diámetro en el punto de inflexión $(\mathrm{cm})$, crpi: crecimiento relativo en el punto de inflexión (\%), tpi: tiempo en el que ocurre el máximo incremento corriente anual del diámetro (en el punto de inflexión de la función, años).

Absolute and comparative values among species ( Na: N. alpina, No: N. obliqua, Nd: $N$. dombeyi) and strata (EI: intermediate, ES: superior) of life parameters (PV) of the diametric growth model. dap $:$ maximum asymptotic diameter $(\mathrm{cm}), \mathrm{cma}$ : mean absolute growth ( $\mathrm{cm}$ year $\left.{ }^{-1}\right), t v$ : life-time (years), dpi: diameter at the inflection point (cm), crpi: relative growth at the inflection point (\%), tpi: time of absolute maximum growth (years).

\begin{tabular}{|c|c|c|c|c|c|c|c|c|c|c|c|c|c|}
\hline \multirow{2}{*}{ PV } & \multicolumn{5}{|c|}{ EI } & \multicolumn{5}{|c|}{ ES } & \multicolumn{3}{|c|}{$\mathrm{EI} / \mathrm{ES}$} \\
\hline & $\mathrm{Na}$ & No & $N d$ & $\mathrm{Na} / \mathrm{Nd}$ & No/Nd & $\mathrm{Na}$ & No & $N d$ & $\mathrm{Na} / \mathrm{Nd}$ & No/Nd & $\mathrm{Na}$ & No & $N d$ \\
\hline$a$ & 0,32 & 0,32 & 0,31 & 1,03 & 1,03 & 0,54 & 0,59 & 0,43 & 1,26 & 1,37 & 0,59 & 0,54 & 0,72 \\
\hline$k$ & 0,009 & 0,008 & 0,008 & 1,13 & 1,00 & 0,013 & 0,009 & 0,013 & 1,00 & 0,69 & 0,69 & 0,89 & 0,62 \\
\hline$c$ & 0,21 & 0,07 & 0,22 & 0,95 & 0,32 & 0,23 & 0,10 & 0,32 & 0,72 & 0,31 & 0,91 & 0,70 & 0,69 \\
\hline$d a p_{m}$ & 70,60 & 62,00 & 74,30 & 0,95 & 0,84 & 88,60 & 74,00 & 100,0 & 0,89 & 0,74 & 0,80 & 0,84 & 0,74 \\
\hline cma & 0,25 & 0,23 & 0,24 & 1,05 & 0,96 & 0,47 & 0,33 & 0,49 & 0,95 & 0,68 & 0,53 & 0,70 & 0,49 \\
\hline tv & 277,0 & 267,0 & 307,0 & 0,90 & 0,87 & 190,0 & 221,0 & 203,0 & 0,94 & 1,09 & 1,46 & 1,21 & 1,51 \\
\hline$d p i$ & 21,00 & 3,60 & 23,70 & 0,88 & 0,15 & 13,30 & 8,90 & 46,10 & 0,29 & 0,19 & 1,58 & 0,40 & 0,51 \\
\hline$d_{p i} / d a p_{m}$ & 0,30 & 0,06 & 0,32 & 0,93 & 0,18 & 0,15 & 0,12 & 0,46 & 0,33 & 0,26 & 2,00 & 0,50 & 0,70 \\
\hline crpi & 4,10 & 11,50 & 3,50 & 1,17 & 3,27 & 5,60 & 10,30 & 4,10 & 1,37 & 2,54 & 0,73 & 1,12 & 0,85 \\
\hline$t p i$ & 27,40 & 9,10 & 32,40 & 0,85 & 0,28 & 20,60 & 10,20 & 29,70 & 0,69 & 0,34 & 1,33 & 0,89 & 1,09 \\
\hline
\end{tabular}

más jóvenes) y $10 \%$ (en los de mayor edad) mayor al del estrato inferior hasta los 155 años, y a partir de esta edad el valor de incremento diamétrico corriente fue muy bajo y similar en ambos estratos sociales (figura 5).

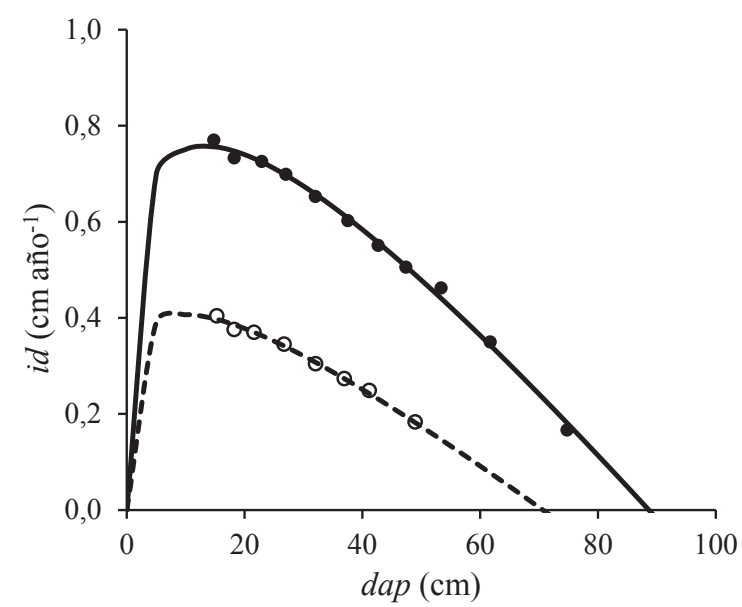

Figura 4. Modelo ajustado y datos promedio del incremento diamétrico corriente $(i d)$ en función del diámetro a la altura del pecho (dap) de Nothofagus alpina de los estratos intermedio (círculo blanco, línea discontinua, $\mathrm{n}=8$ ) y superior (círculo negro, línea continua, $\mathrm{n}=11$ ).

Adjusted model and mean data of current diametric increase (id) as a function of diameter at breast height (dap) of N. alpina within the intermediate (white circle, dashed line, $\mathrm{n}=8$ ) and superior strata (black circle, solid line, $\mathrm{n}=11$ ).
La comparación entre especies. Las diferencias de crecimiento diamétrico entre especies son pequeñas a juzgar por la interpretación de los modelos. Sin embargo, existen discrepancias interespecíficas en función de la posición social. En las clases de mayor edad, $N$. dombeyi presentó la mayor diferencia de crecimiento entre estratos, mientras que, en contraposición, $N$. obliqua presentó la menor diferencia; $N$. alpina exhibió una diferencia intermedia (figuras 5 y 6). En el estrato inferior, $N$. dombeyi superó a $N$. obliqua a partir de los 75 años $(N$. dombeyi $=26,5$ y $N$. obliqua $=26,4 \mathrm{~cm}$ dap ). A los 200 años, $N$. alpina $y$ $N$. dombeyi alcanzaron, en promedio, $55,4 \mathrm{~cm}$ dap mientras que $N$. obliqua $48,7 \mathrm{~cm}$ de dap. En el estrato superior $N$. dombeyi exhibió el comparativo menor dap promedio hasta 10 años $(N$. alpina $=4,7 ; N$. obliqua $=4,9$ y $N$. dombe$y i=4,5 \mathrm{~cm}$ dap $)$. Posteriormente, $N$. dombeyi superó en tamaño a $N$. obliqua a partir de los 15 años ( $N$. dombeyi $=7,9$ y $N$. obliqua $=7,4 \mathrm{~cm}$ dap) y a $N$. alpina a partir de los 55 años $(N$. dombeyi $=37,2$ y $N$. alpina $=36,9 \mathrm{~cm}$ dap $)$. A los 200 años, $N$. dombeyi alcanzó 89,3; $N$. alpina 80,1 y N. obliqua $60,5 \mathrm{~cm}$ dap (figuras 5 y 6 ).

En el estrato inferior, aunque $N$. dombeyi exhibió a los 25 años el valor menor de incremento diamétrico corriente en comparación con el de las demás especies, las diferencias interespecíficas fueron escasas $(N$. alpina $=0,41$; $N$. obliqua $=0,38$ y $N$. dombeyi $=0,37 \mathrm{~cm}^{2}$ ano $\left.{ }^{-1}\right)$. Posteriormente, esta especie superó a $N$. obliqua a los 30 años $\left(N\right.$. dombeyi $=0,38$ y $N$. obliqua $=0,37 \mathrm{~cm}$ año $\left.^{-1}\right)$ y a $N$. alpina a los 80 años $(N$. dombeyi $=0,33$ y $N$. alpina $=$ 

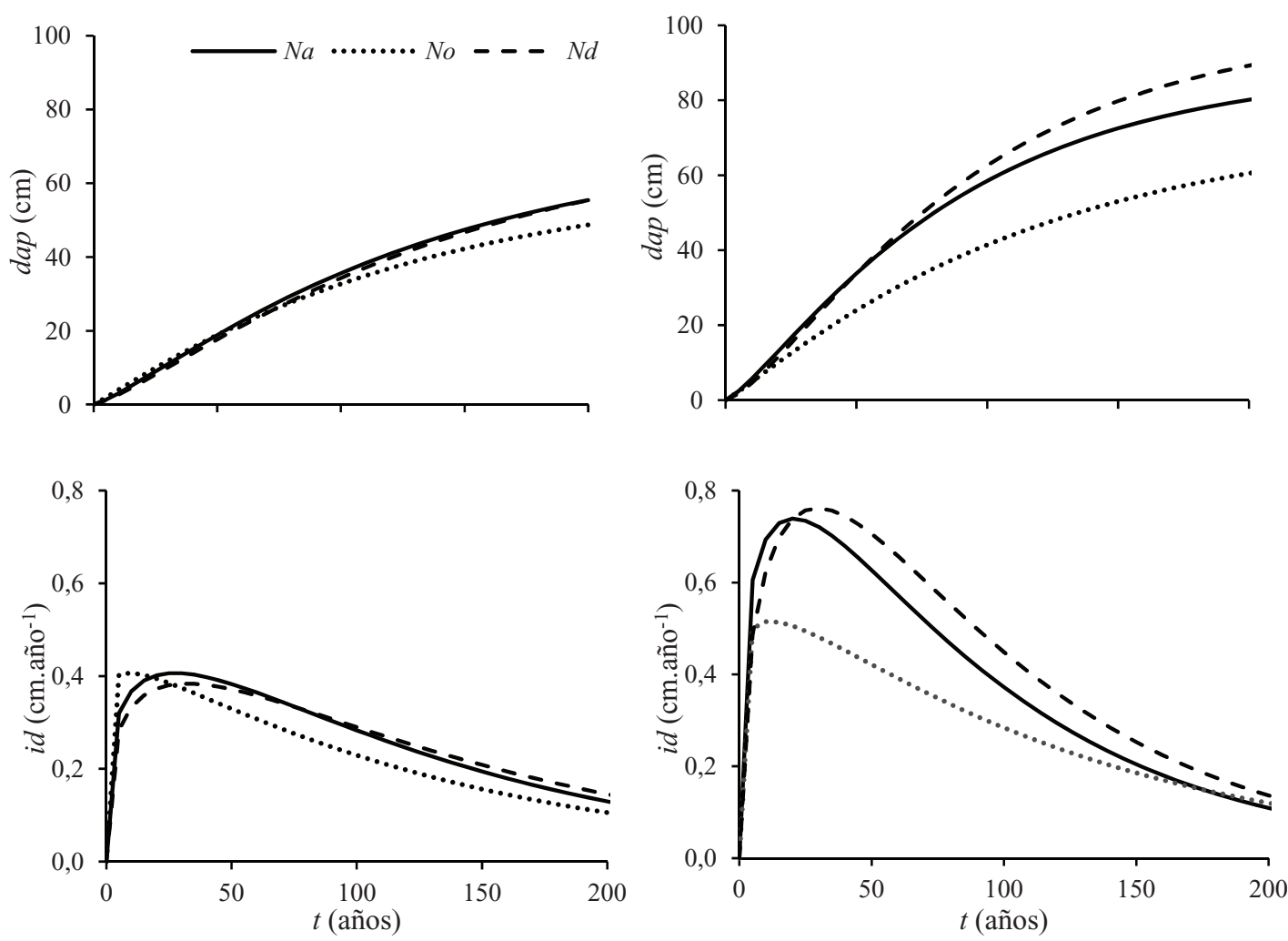

Figura 5. Rendimiento (dap) e incremento corriente diamétricos (id) de Nothofagus alpina (Na), N. obliqua (No) y N. dombeyi (Nd) a lo largo del tiempo $(t)$ de los estratos intermedio (izquierda) y superior (derecha).

Yield (dap) and current increment of diameter (id) in $N$. alpina $(\mathrm{Na}), N$. obliqua $(\mathrm{No})$ and $N$. dombeyi $(\mathrm{Nd})$ along time $(t)$ within the intermediate (left) and superior strata (right).

$0,32 \mathrm{~cm}$ año $\left.{ }^{-1}\right)$. A los 200 años, $N$. dombeyi alcanzó 0,15 $N$. alpina 0,13 y $N$. obliqua $0,11 \mathrm{~cm}$ año-1 $i d$. En el estrato superior, $N$. dombeyi exhibió el valor menor de incremento diamétrico corriente en comparación con el de las otras especies hasta los 5 años $(N$. alpina $=0,60, N$. obliqua $=0,50$ y $N$. dombeyi $=0,49 \mathrm{~cm}$ año $\left.\mathrm{o}^{-1}\right)$. Posteriormente, $N$. dombeyi superó a $N$. obliqua a los 10 años $(N$. dombeyi $=0,62$ y $N$. obliqua $=0,51 \mathrm{~cm}$ año $^{-1}$ ) y a $N$. alpina a los 25 años $\left(N\right.$. dombeyi $=0,76$ y $N$. alpina $=0,73 \mathrm{~cm}^{2}$ año $\left.{ }^{-1}\right)$. A los 200 años, $N$. dombeyi alcanzó un valor de incremento diamétrico corriente igual a 0,$14 ; N$. obliqua 0,12 y $N$. alpina $0,11 \mathrm{~cm}^{2}$ ño- ${ }^{-1}$ (figuras 5 y 6 ).

Para $N$. alpina, el modelo de crecimiento predijo valores de $d a p_{m}, c m a, t v, d p i$, crpi y tpi en el estrato inferior iguales a 70,6 cm, 0,25 cm año ${ }^{-1}, 277$ años, $21,0 \mathrm{~cm}, 4,1 \%$ y 27,4 años, respectivamente, y en el estrato superior iguales a $88,6 \mathrm{~cm}, 0,47 \mathrm{~cm}_{\text {año }}{ }^{-1}, 190$ años, $13,3 \mathrm{~cm}, 5,6 \%$ y 20,6 años, respectivamente. La comparación de estos parámetros entre especies dentro de los estratos mostró que, en el estrato inferior, los valores de $d p i, c m a$ y $t v$ de $N$. alpina y $N$. obliqua fueron similares a los de $N$. dombeyi. Los valores de $d p i, d p i / d a p_{m}$ y tpi de $N$. alpina fueron equivalentes a los de $N$. dombeyi, mientras que los de $N$. obliqua fueron más bajos. El valor de crpi de $N$. obliqua fue mayor al de N. dombeyi. En el estrato superior, los valores de $d a p_{m}, c m a$ y $t v$ de $N$. dombeyi fueron similares a los de $N$. alpina y $N$. obliqua, mientras que los de dpi, dpi/dap ${ }_{m}$ y tpi fueron mayores y el valor de crpi fue menor (cuadro 2).

La comparación de los parámetros de vida entre estratos dentro de las especies mostró que en $N$. alpina, $N$. obliqua y $N$. dombeyi, los árboles del estrato inferior exhibieron valores menores de $d a p_{m}$ y $c m a$ y mayores de $t$. En cambio, los valores de $d p i$ y $d p i / d a p_{m}$ del estrato inferior de $N$. alpina fueron más altos que los del estrato superior, mientras que los valores para el estrato inferior de las demás especies fueron más bajos que en el estrato superior. El valor de los parámetros de vida del estrato intermedio de $N$. alpina fue compatible con árboles que exhiben crecimiento menor durante períodos más breves; dap ${ }_{m}, \mathrm{cma}$ y crpi fueron menores, y tv fue mayor (cuadro 2).

\section{DISCUSIÓN}

El diámetro del fuste de los árboles intermedios en las tres especies aumentó a menor velocidad que el de los superiores. Por ejemplo, el incremento corriente medio de $N$. alpina del estrato intermedio, según la base de datos, y máximo, según el modelo de crecimiento, fue práctica- 

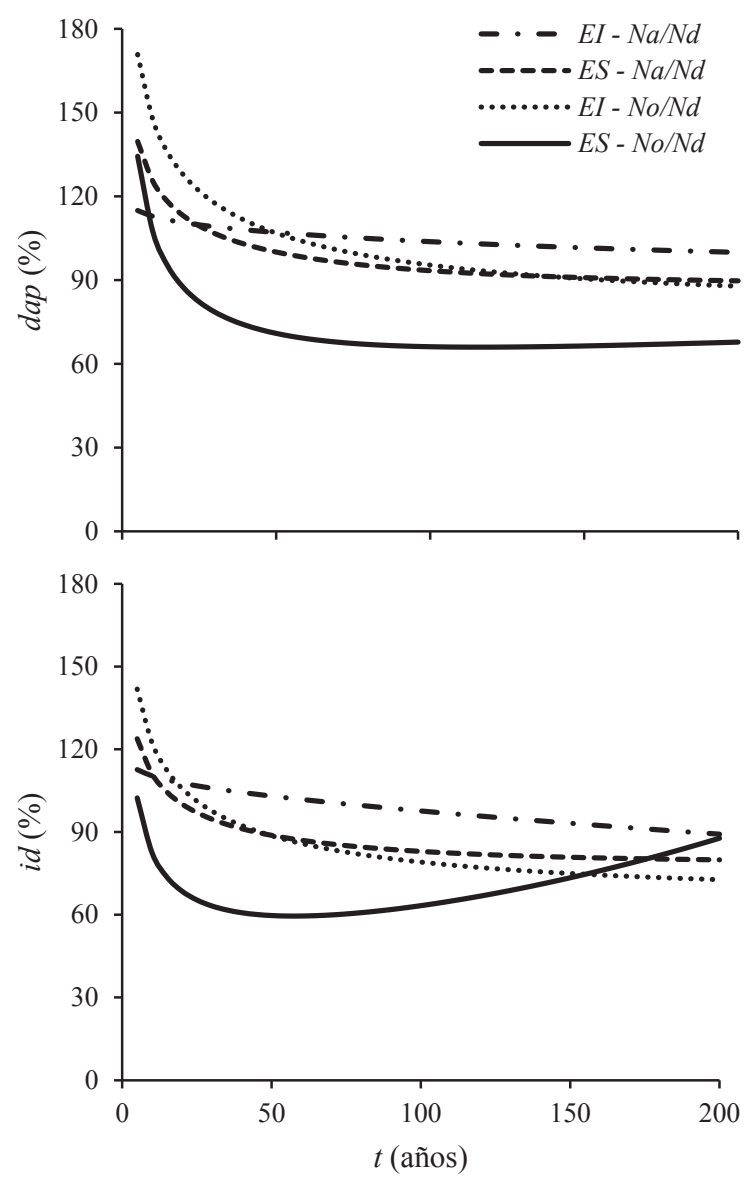

Figura 6. Valor relativo de rendimiento (dap) e incremento corriente diamétricos (id) de Nothofagus alpina $(\mathrm{Na})$ y $N$. obliqua $(N o)$ con relación a $N$. dombeyi $(N d)$ a lo largo del tiempo $(t)$ de los estratos intermedio (EI) y superior (ES).

Relative value of yield (dap) and current increment of diameter (id) in N. alpina $(\mathrm{Na})$ and $N$. obliqua $(\mathrm{No})$ relative to $N$. dombeyi $(\mathrm{Nd})$ along time $(t)$ within the intermediate (EI).

mente la mitad al del estrato superior. En comparación con el estrato superior, el intermedio presentó en las clases de edad menor un incremento corriente y rendimiento $93 \%$ menor, y en las de edad mayor un rendimiento $46 \%$ menor y un incremento corriente $15 \%$ menor. El menor crecimiento de los árboles intermedios se asociaría a que recibieron una menor cantidad de luz y con una composición espectral fotosintéticamente menos óptima, debido a la mayor reflexión y absorción por parte de los árboles dominantes (Pallardy 2007).

Aunque los árboles del estrato intermedio crecieron más lentamente, la culminación del incremento corriente anual fue similar a la de los árboles del estrato superior. El mantenimiento por parte de los árboles subordinados de una forma de crecimiento equivalente al de los dominantes y codominantes, indicaría la existencia de un patrón de crecimiento común independientemente de las condicio- nes de luz (Chauchard et al. 2001, Chauchard y Sbrancia 2003). La escasa diferencia de crecimiento a lo largo del tiempo dentro de cada especie y entre estratos, se podría deber a que árboles dominantes alcanzaron el valor asintótico de tamaño y luego disminuyeron su crecimiento. Sin embargo, la limitación impuesta por el diámetro máximo de los individuos medidos también podría contribuir a explicar este resultado.

El crecimiento de $N$. alpina, comparado con el de $N$. dombeyi, fue similar en todas las clases de edad para el estrato intermedio, y menor en las clases de mayor edad para el estrato superior. Sin embargo, el desempeño de esta especie fue muy adecuado en ambos estratos, que se asociaría a la mayor tolerancia a la sombra (percibida en el estrato intermedio) y la positiva respuesta al aumento de la luz (en el superior). El mayor carácter umbrófilo de los renovales de $N$. alpina fue documentado previamente. Grosse (1988) y Donoso et al. (2013) hallaron en rodales mixtos implantados, una mayor sobrevivencia y crecimiento de estos renovales en condiciones de menor luminosidad asociados a claros pequeños del dosel. En ecosistemas naturales mixtos, Dezzotti et al. (2004) y Sola et al. (2015) documentaron que los renovales de $N$. alpina constituyeron la "regeneración avanzada" en rodales vírgenes, y presentaron mayor crecimiento en los bordes de los claros en rodales luego de la implementación de cortas de selección. Estos autores propusieron además que este sistema silvicultural no mantendría la composición relativa originalmente equilibrada, debido a la menor competitividad de los renovales de $N$. alpina asociado al aumento de la radiación en el piso del bosque. La mayor tolerancia a la sombra de los renovales de $N$. alpina se asocia a menores valores de respiración y punto de compensación de luz (Read y Hill 1985).

Los individuos maduros de $N$. dombeyi del estrato superior crecieron a mayor velocidad que los de las demás especies; este resultado es compatible con el conocimiento acerca de su alto potencial de crecimiento (Tuley 1980, Müller-Using y Schlegel 1981, Chauchard et al. 2001). Sin embargo, los individuos jóvenes de $N$. dombeyi exhibieron un crecimiento similar al de $N$. alpina y $N$. obliqua; esto podría deberse a que el tamaño de los claros no fue el adecuado para que esta especie heliófila exprese su máximo potencial. Müller-Using y Schlegel (1981), Donoso (1993) y Dezzotti et al. (2004) adjudicaron el menor crecimiento temprano de $N$. dombeyi (e.g., durante los primeros dos años), al significativo menor peso y reserva de nutrientes de la semilla. A lo largo de la distribución geográfica, el peso de la semilla es hasta $4,4 \mathrm{mg}$, mientras que la de $N$. obliqua es hasta $21,3 \mathrm{mg}$ y la de $N$. alpina $24,4 \mathrm{mg}$ (Dezzotti et al. 2016). El papel de esta reserva sobre la variación del diámetro perdería importancia, en favor del de la luz, en etapas posteriores (Müller-Using y Schlegel 1981, Donoso et al. 2013).

El modelo permitió representar adecuadamente la variación del diámetro del fuste de $N$. alpina, a juzgar por 
el comportamiento del valor de los parámetros dentro de límites biológicamente esperados. El modelo además se conformó con variables sencillas de medir y funciones simples, que potencian su utilidad sin afectar sustancialmente la precisión (Crecente-Campo et al. 2010, Vanclay 2010, Ivancich et al. 2014). Esta precisión debe ser compatible con la complejidad y variabilidad estructural de los sistemas boscosos, particularmente, la que ocurre en los naturales sin manejo silvicultural (Vanclay 1994). Sin embargo este modelo, y el de N. obliqua (Chauchard y Sbrancia 2003) y N. dombeyi (Chauchard et al. 2001), exhiben ciertas limitaciones. Por un lado, se ajustó utilizando valores medios de las variables para cada clase diamétrica, por lo que solo predice el valor promedio más esperado. Por otro lado, no consideró la densidad del rodal, y por lo tanto las predicciones se deben tomar con prudencia en condiciones de alta densidad del rodal.

\section{CONCLUSIONES}

El modelo von Bertalanffy-Richards desarrollado, describe adecuadamente el crecimiento diamétrico de $N$. alpi$n a$, a través de variables de medición y funciones matemáticas sencillas. En el marco de la silvicultura, este modelo permite predecir el incremento corriente y el rendimiento diamétricos del estrato intermedio y superior para periodos de hasta una década. En el marco de la ecología, el modelo posibilita inferir aspectos asociados a la tolerancia a la sombra de esta especie, e interpretarlos conjuntamente con los de $N$. obliqua y $N$. dombeyi. La comparación revela, por un lado, un comportamiento ecológico común de estas especies de Nothofagus asociado a la demanda de luz $\mathrm{y}$, por otro lado, divergencias compatibles con el mayor carácter umbrófilo de $N$. alpina y heliófilo de $N$. dombeyi que resulta en pequeñas diferencias en las magnitudes de crecimiento en diámetro. Estos resultados contribuyen a aumentar la comprensión del funcionamiento de esta valiosa comunidad subantártica del noroeste de la Patagonia argentina, en el contexto de su conservación y manejo sostenible.

\section{REFERENCIAS}

APN (Administración de Parques Nacionales, AR). 2012. Plan de gestión del Parque Nacional Lanín: caracterización y diagnóstico. Equipo de Planificación del Parque Nacional Lanín, Administración de Parques Nacionales, Buenos Aires. $225 \mathrm{p}$.

Attis Beltrán H, L Chauchard, G Martínez Pastur. 2015. Curvas preliminares de índice de sitio para bosques puros y mixtos de Nothofagus alpina y Nothofagus obliqua en la Patagonia argentina. Bosque 36(2): 275-285.

Cabrera A. 1971. Fitogeografía de la República Argentina. Boletín de la Sociedad Argentina de Botánica 14: 1-42.

Chauchard L. 1991. Modelos de crecimiento individual del raulí (Nothofagus alpina (Poepp. et Endl.) Oerst.). In Actas VI Jornadas sobre inventario, modelos de producción y creci- miento forestales. Eldorado, Argentina. p. 246-276.

Chauchard L, R Sbrancia, A Rabino, M González Peñalba, L Maresca. 2001. Modelos de crecimiento diamétrico para Nothofagus dombeyi. Bosque 22(2): 53-68.

Chauchard L, R Sbrancia. 2003. Modelos de crecimiento diamétrico para Nothofagus obliqua. Bosque 24(3): 3-16.

Crecente-Campo F, P Soares, M Tomé, U Diéguez-Aranda. 2010. Modelling annual individual-tree growth and mortality of Scots pine with data obtained at irregular measurement intervals and containing missing observations. Forest Ecology and Management 260: 1695-1974.

Del Valle H. 1998. Patagonian soils: a regional synthesis. Ecología Austral 8: 103-123.

Dezzotti A, R Sbrancia, D Roat, M Rodríguez-Arias, A Parisi. 2004. Colonización y crecimiento de renovales de Nothofagus después de cortas selectivas de un rodal en la Patagonia, Argentina. Investigación Agraria Serie Forestal 13(2): 329-337.

Dezzotti A, M Manzoni, R Sbrancia. 2016. Producción, almacenamiento en el suelo y viabilidad de las semillas de Nothofagus dombeyi, N. obliqua y N. alpina en un bosque templado del noroeste de la Patagonia argentina. Revista de la Facultad de Agronomía (La Plata) 115(2): 155-172.

Donoso C. 1993. Bosques templados de Chile y Argentina: variación, estructura y dinámica. Santiago, Chile. Editorial Universitaria. $455 \mathrm{p}$.

Donoso P, D Soto, R Coopman, S Rodríguez-Bertos. 2013. Early performance of planted Nothofagus dombeyi and Nothofagus alpina in response to light availability and gap size in a high-graded forest in the south-central Andes of Chile. Bosque 34(1): 23-32.

Grosse H. 1988. Crecimiento de plantaciones de raulí y roble bajo dosel en dependencia del grado de luminosidad y fertilización. Ciencia e Investigación Forestal 2(5): 13-30.

Ivancich H, G Martínez Pastur, MV Lencinas, JM Cellini, P Peri P. 2014 Proposals for Nothofagus antarctica diameter growth estimation: simple vs. global models. Journal of Forest Science 60(8): 307-317.

Lara A, P Rutherford, C Montory, D Bran, A Pérez, S Clayton, J Ayesa, D Barrios, M Gross, G Iglesias. 1999. Vegetación de la eco-región de los Bosques Valdivianos. Boletín Técnico Fundación Vida Silvestre 51: 1-29.

Melo O, R Vargas. 2003. Evaluación ecológica y silvicultural de ecosistemas boscosos. Ibagué, Colombia. Universidad de Tolima. $235 \mathrm{p}$.

Müller-Using B, F Schlegel. 1981. The development of seedlings of Chilean Nothofagus species in a shaded area. Plant Research and Development 12: 152-184.

Oliver CD, BC Larson. 1996. Forest stand dynamics. New York, USA. Wiley. 544 p.

Pallardy S. 2007. Physiology of woody plants. San Diego, USA. Academic Press. 464 p.

Paruelo J, A Beltrán, E Jobbágy, O Sala, R Golluscio. 1998. The climate in Patagonia: general patterns and controls on biotic processes. Ecología Austral 8: 85-101.

Ramírez C, C San Martín, A Oyarzún, H Figueroa. 1997. Morpho-ecological study of the South American species of the genus Nothofagus. Plant Ecology 130: 101-109.

Read J, R Hill. 1985. Photosynthetic responses to light of Australian and Chilean species of Nothofagus and their relevance to the rain forest dynamics. New Phytologist 101: 731-743. 
Richards F. 1959. A flexible growth function for empirical use. The Journal of Experimental Botany 10(29): 290-300.

Smith D, B Larson, M Kelty, P Ashton. 1996. The practice of silviculture: applied forest ecology. New York, USA. Wiley. 560 p.

Sola G, H Attis Beltrán, L Chauchard, L Gallo. 2015. Efecto del manejo silvicultural sobre la regeneración de un bosque de Nothofagus dombeyi, N. alpina y N. obliqua en la Reserva Nacional Lanín (Argentina). Bosque 36(1): 113-120.

Stokes M, T Smiley. 1996. An introduction to tree-ring dating. Tucson, USA. University of Arizona Press. 73 p.

Tilman D. 1988. Plant strategies and the dynamic and structure of plant communities. Princeton, USA. Princeton University Press. 360 p.

Tomé J, M Tomé, S Barreiro, J Amaral. 2006. Age independent difference equations for modelling tree and stand growth.
Canadian Journal of Forest Research 36: 1621-1630.

Tuley G. 1980. Nothofagus in Britain. Forest Record 122: 1-26.

Vanclay J. 1994. Modelling forest growth and yield: applications to mixed tropical forests. Wallingford, USA. CAB International. 312 p.

Vanclay J. 2010. Robust relationships for simple plantation growth models based on sparse data. Forest Ecology and Management 259: 1050-1054.

Veblen T, C Donoso, T Kitzberger, A Rebertus. 1996. Ecology of southern Chilean and Argentinean Nothofagus forests. In Veblen T, R Hill, J Read eds. The ecology and biogeography of Nothofagus forests. New Haven, USA. Yale University Press. p. 293-353.

Weinberger P, C Ramírez. 2001. Microclima y regeneración natural de raulí, roble y coigüe (Nothofagus alpina, N. obliqua y N. dombeyi). Bosque 22(1): 11-26.

Recibido: 29.06 .17

Aceptado: 16.01 .18 
